\title{
Efectos Morfológicos y Mecánicos en Ratas Sprague Dawley Sometidas a Ciclos de Hipoxia
}

\author{
Morphological and Mechanical Effects in Sprague Dawley Rats Subjected to Hypoxia Cycles
}

\author{
Daniel Conei ${ }^{1,2}$; Mariano del Sol${ }^{1}$; Rodrigo Muñoz Cofré1 ${ }^{1}$ Máximo Escobar Cabello ; \\ Héctor Saavedra P. ${ }^{4}$; Emilio A. Herrera ${ }^{5}$ \& Claudio M. García-Herrera ${ }^{4}$
}

CONEI, D.; DEL SOL, M.; MUÑOZ, R.; ESCOBAR, C. M.; SAAVEDRA, H.; HERRERA, E. A. \& GARCÍA-HERRERA, C. M. Efectos morfológicos y mecánicos en ratas Sprague Dawley sometidas a ciclos de hipoxia. Int. J. Morphol., 37(3):908-911, 2019.

RESUMEN: Periodos extensos de hipoxia provocan cambios adaptativos que permiten responder a las demandas impuestas por el ambiente. Sin embargo, existen casos donde esta exposición es intermitente, como es el caso de los trabajadores en zonas andinas. El objetivo de esta comunicación fue comprobar los efectos morfológicos y mecánicos en diafragma y pulmones de ratas sometidas a la hipoxia intermitente. Se utilizaron 4 ratas Sprague Dawley de 6 meses de edad. Dos ratas fueron sometidas a 10 ciclos de hipoxia hipobárica intermitente (HHI) de 96 h ( 428 torr; $\mathrm{PO}_{2} 90 \mathrm{~mm} \mathrm{Hg}$ ), seguidos de 96 h de normoxia normobárica, durante 80 días. Se realizaron pruebas tracción uniaxial y de tinción con HematoxilinaEosina y Picrosirius red de Junqueira. Al comparar las curvas de los diafragmas, los sometidos a hipoxia reducen levemente su esfuerzo respecto a la condición de normoxia, en el tejido pulmonar la hipoxia afecta negativamente su resistencia, estas muestran una pendiente menor respecto a las normóxicas. En el análisis histológico, el parénquima pulmonar presentó menor cantidad de vasos sanguíneos y celularidad, como una mayor fracción de área de los espacios alveolares y cantidad de colágeno total en el grupo HHI. En el diafragma, el grupo HHI presentó menor cantidad de miocitos distribuidos irregularmente y de colágeno total. En conclusión, los principales hallazgos indican que el diafragma y el tejido pulmonar sometido a HHI sufren cambios estructurales, que se traducen en una disminución en su capacidad de resistencia tensil.

PALABRAS CLAVE: Hipoxia; Diafragma; Pulmones; Tensión.

\section{INTRODUCCIÓN}

La necesidad del intercambio gaseoso se genera a partir del gasto metabólico que le implica al organismo mantenerse en equilibrio. Este proceso, a su vez, establece el nivel de ventilación pulmonar necesaria para cubrir esta demanda (Mortola, 2019). Este hecho ocurre a nivel del mar, sin embargo, al aumentar la altitud disminuye la presión atmosférica, por tanto, cuando respiramos la presión del aire al interior de los pulmones con $\mathrm{PaO}_{2}$ son menores, cae el aporte de oxígeno a la sangre y a los tejidos. Esto se conoce como hipoxia (Steva et al., 2009).

En este contexto, se hace necesario el correcto funcionamiento del sistema respiratorio. El cual contempla un aumento de la contracción de los músculos inspiratorios, los que generan gradientes de presión, y el intercambio de gases en los alvéolos (Adaos et al., 2017). Complementariamente, las respuestas vasculares pulmonares aseguran la oxigenación adecuada de la sangre en respuesta a las bajas tensiones de oxígeno, respuestas que, a su vez son iniciadas en procesos agudos de hipoxia (Mortola).
La hipoxia puede ser intermitente (HI) en el caso de trabajadores andinos, siendo un modelo único en donde los períodos de exposición a bajas concentraciones de $\mathrm{O}_{2}$ se intercalan con estadías a nivel del mar. Situación distinta a la ocurrida en eventos agudos (turismo y apnea del sueño) o crónicos como la residencia en altura, donde la hipoxia puede ser acotada o permanente, respectivamente (Herrera et al., 2016).

Frente a esta situación, existe un mecanismo fisiológico denominado vasoconstricción pulmonar hipóxica, el cual restringe el flujo sanguíneo a los alvéolos menos oxigenados y lo redirige hacia los mejor oxigenados. Sin embargo, cuando esta respuesta se mantiene por un largo tiempo genera cambios morfofuncionales, dentro de los que destacan un aumento de la vascularización pulmonar y en ocasiones una disminución del número de alvéolos (Urrutia \& Aragonés, 2018).

Paralelamente, como todo músculo estriado, el diafragma puede verse modificado frente a factores de estrés

\footnotetext{
${ }^{1}$ Programa de Doctorado en Ciencias Morfológicas, Universidad de La Frontera, Temuco, Chile.

${ }^{2}$ Departamento de Ciencias Morfológicas, Facultad de Medicina y Ciencia, Universidad San Sebastián, Puerto Montt, Chile. ${ }^{3}$ Programa de Función Disfunción Ventilatoria, Departamento de Kinesiología, Universidad Católica del Maule, Talca, Chile. ${ }^{4}$ Departamento de Ingeniería Mecánica, Facultad de Ingeniería, Universidad de Santiago de Chile, Santiago, Chile.

${ }^{5}$ Programa de Fisiopatología, Facultad de Medicina, Instituto de Ciencias Biomédicas, Universidad de Chile, Santiago, Chile.
} 
fisiológicos y/o fisiopatológicos, con el propósito de mantener la homeostasis respiratoria (Lewis \& O'Halloran, 2016). Considerando que el diafragma está sometido a cargas cíclicas producidas por la respiración, es relevante estudiar el efecto de los ciclos de hipoxia en él. Es común que bajo diferentes condiciones de hipoxia el diafragma tienda a disminuir su fuerza, pero aumente su resistencia (McMorrow et al., 2011). Esto involucra adaptaciones estructurales y funcionales, las cuales incluyen, disminución de la respiración mitocondrial, activación de la señalización de atrofia muscular y aumento de la proteólisis (Lewis \& O’Halloran; Panisello et al., 2008).

El músculo esquelético está compuesto por agua, colágeno y tejido adiposo, cubierto por tejido conectivo otorgándole propiedades viscoelásticas derivadas de su estructura. Debido a su importancia, se han implementado variados modelos para la comprensión del sistema musculoesquelético. Así, la prueba de estrés por estiramiento aportaría información de la capacidad de la capacidad tensíl del músculo (Kuthe \& Uddanwadiker, 2014), información que en este caso sería relevante considerando los daños estructurales que se han reportado en el diafragma sometido a HI.

El objetivo de esta comunicación fue comprobar los efectos morfológicos y mecánicos en diafragma y pulmones de ratas sometidas a $\mathrm{HI}$.

\section{MATERIAL Y MÉTODO}

Se utilizaron 4 ratas Sprague Dawley de 6 meses de edad. Dos ratas fueron sometidas a 10 ciclos de hipoxia hipobárica intermitente (HHI) de 96 h ( 428 torr; $\mathrm{PO}_{2} 90$ $\mathrm{mm} \mathrm{Hg}$ ), seguidos de $96 \mathrm{~h}$ de normoxia normobárica, durante 80 días. Las dos ratas en normoxia, se mantuvieron a $22^{\circ} \mathrm{C}$ con ciclo de luz/oscuridad de $12 \mathrm{~h}$ cada uno. Ambos grupos recibieron la misma cantidad de alimento y agua a diario (15 g de comida estándar en pellets; agua ad libitum). Después de los ciclos de exposición, se realizó eutanasia con pentobarbital $\left(50 \mathrm{mg} / \mathrm{kg}^{-1}\right.$ intraperitoneal). Se extrajeron segmentos de tejido pulmonar y diafragma, a los cuales, se les realizó pruebas de tracción uniaxial, tinción con Hematoxilina-Eosina y Picrosirius red de Junqueira.

Los ensayos de tracción fueron realizados en una máquina de ensayos universal (Instron 3342®), las muestras fueron cortadas en forma rectangular, se registró el área y espesor, con el propósito estimar carga y estiramiento. Posteriormente, los tejidos se posicionaron en un sistema de montaje para tejidos blandos, donde estuvieron sumergida en Krebs a una temperatura de $37^{\circ} \mathrm{C}$ durante todo el ensayo. La tracción se realizó con una velocidad de $1 \mathrm{~mm} / \mathrm{min}$, la información de carga y desplazamiento se obtuvo con una precisión de 0,01 $\mathrm{N}$ y $1 \mu \mathrm{m}$, respectivamente.

Se realizaron 20 cortes transversales de $5 \mu \mathrm{m}$ de espesor con micrótomo Leica (RM2255) y se montaron en portaobjetos xilanizados. Posteriormente, se realizó tinción con H-E y Picrosirius red de Junqueira para la marcación de colágeno. Las muestras fueron fotografiadas con un microscopio de luz Leica (DM750) con cámara digital HD Leica (ICC50 HD) con el objetivo 100x. Se analizaron 5 campos al azar de cada corte histológico. Las imágenes fueron analizadas con el programa ImageJ 1.8.0 (NIH, E.E.U.U.), utilizando la herramienta Threshold, se cuantificaron las fracciones de área de: espacios alvéolares, celularidad y colágeno presente en alveolos; miocitos y colágeno en diafragma.

\section{RESULTADOS}

Ensayos de tracción. En las Figs. 1 y 2, se observa que a bajas deformaciones el comportamiento presenta mínima desviación, sin embargo, a medida que aumenta la deformación, la dispersión aumenta. Al comparar las curvas de los diafragmas, los sometidos a hipoxia reducen levemente su esfuerzo respecto a la condición de normoxia. En contraste a estos resultados, en el tejido pulmonar se observa que la hipoxia afecta negativamente su resistencia, estas muestran una pendiente menor respecto a las normoxicas. Comparando las muestras de tejidos pulmonares respecto a las de diafragma se observa que estas últimas son más resistentes que las de pulmón, debido a que su magnitud de esfuerzo está sobre los 500 mega pascales $(\mathrm{MPa})$, versus la del tejido pulmonar que llega a los $50 \mathrm{MPa}$, sin embargo, este último es más deformable que los diafragmas analizados.

Análisis histológico. En el parénquima pulmonar del grupo normoxia se observó una mayor cantidad de vasos sanguíneos en comparación al grupo HHI. La fracción de área de los espacios alveolares fue de $28,76 \pm 0,69 \%$ para el grupo normoxia y de $32,13 \pm 0,58 \%$ para grupo HHI. La cuantificación de células totales a nivel alveolar fue mayor en el grupo normoxia $(84,46 \pm 2,18 \%)$ en relación al grupo de HHI $(75,53 \pm 1,92 \%)$ (Fig. 3A y 3C). La distribución de colágeno tipo I y III fue diferente, el grupo normoxia presentó $0,37 \pm 0,12 \%$ de fibras colágenas y el grupo HHI 1,56 $\pm 0,41 \%$ (Fig. 3E y 3G). En el diafragma la cantidad relativa de miocitos por campo en el grupo normoxia fue de $84,37 \pm 1,84 \%$ y de $75,25 \pm 2,80 \%$ en el grupo HHI (Fig. 3B y 3D). Este último presentó una distribución irregular de las fibras musculares. Respecto al colágeno, el grupo normoxia presentó un $14,19 \pm 2,36 \%$ de fibras por campo, en comparación al $8,31 \pm 1,72 \%$ del grupo HHI (Fig. $3 \mathrm{~F}$ y $3 \mathrm{H}$ ). 


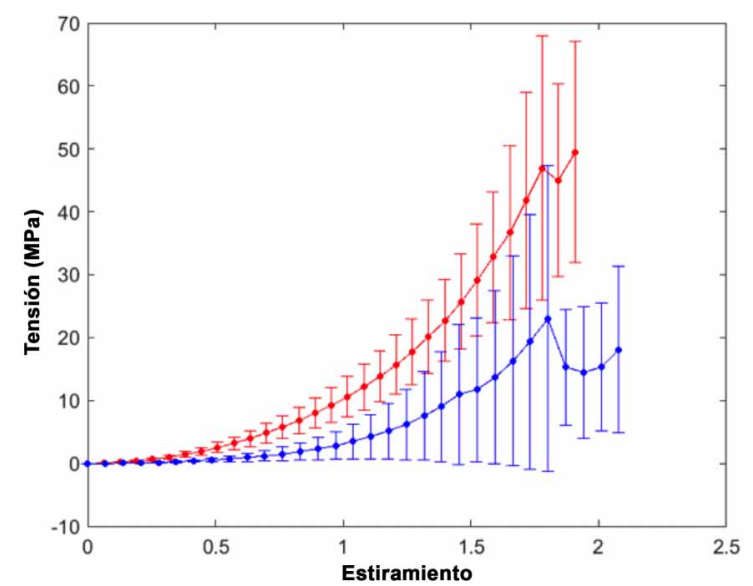

Fig 1. Resultados de ensayo de tracción uniaxial de diafragmas en ratas HHI y normóxicas. MPa: megapascales; normoic: normoxia; hypoxic: hipoxia.

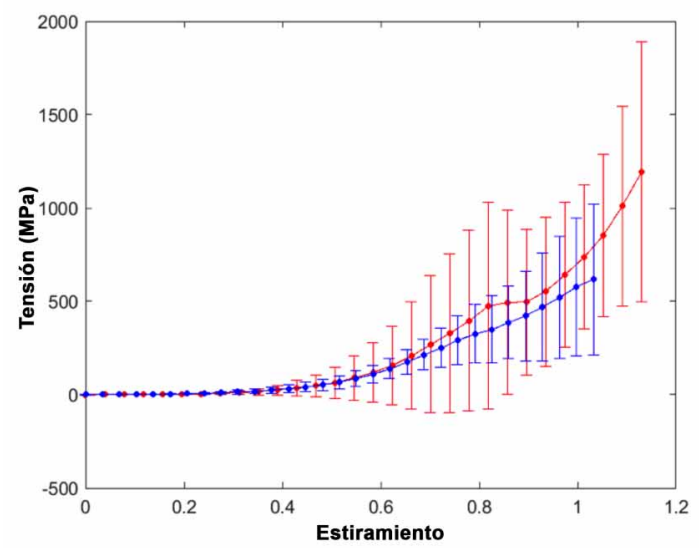

Fig 2. Resultados de ensayo de tracción uniaxial de pulmones en ratas HHI y normóxicas. MPa: mega-pascales; normoic: normoxia; hypoxic: hipoxia.

\section{DISCUSIÓN}

El objetivo de esta comunicación fue comprobar los cambios asociados a la HHI en el diafragma y tejido pulmonar de ratas. Los hallazgos indican que ambos sufren cambios estructurales, los que se traducen en una disminución en su capacidad de resistir tensión. Situación no menor, debido a que el sistema respiratorio tiene una función vital de aquí su funcionamiento rítmico y continuo a lo largo de la vida (Adaos et al.).

En un ensayo de Zhu et al. (2005), la función expresada a través de la relación fuerza-frecuencia (in vitro), disminuyó significativamente $(p<0,001)$ en ratas sometidas a 60 min de hipoxia versus ratas sometidas a $60 \mathrm{~min}$ de hiperoxia. Paralelamente, la resistencia a la fatiga también fue significativa-mente menor en las ratas sometidas a hipoxia $(\mathrm{p}<0,05)$. Los datos de tensión obtenidos en el estudio pueden contribuir a complementar lo conocido, dado que el diafragma del grupo con HHI, con menos carga alcanzó menos tensión, en cambio, el grupo en normoxia con una carga mayor alcanzó una mayor tensión.

Panisello et al. (2008) sometieron a ratas a HHI (22 días; 4h/día, 5 días/semana) y observaron un aumento significativo en la densidad capilar de las ratas con HHI (736 capilares $/ \mathrm{mm}^{2}$ ) versus el grupo control $\left(610\right.$ capilares $\left./ \mathrm{mm}^{2}\right)$. Además, estos mismos autores, observaron una reducción del 13 $\%$ y $7 \%$ en el área y perímetro, respectivamente, de fibras lentas de las ratas con HHI en relación al control. Esto indicaría que las fibras lentas serían más sensibles a $\mathrm{HHI}$ que los dos tipos de fibras rápidas. Los resultados encontrados en esta comunicación concuerdan con los valores reportados por Panisello et al., específicamente la cantidad de miocitos relativa por campo fue de $84,37 \pm 1,84 \%$ en el grupo normoxia y de

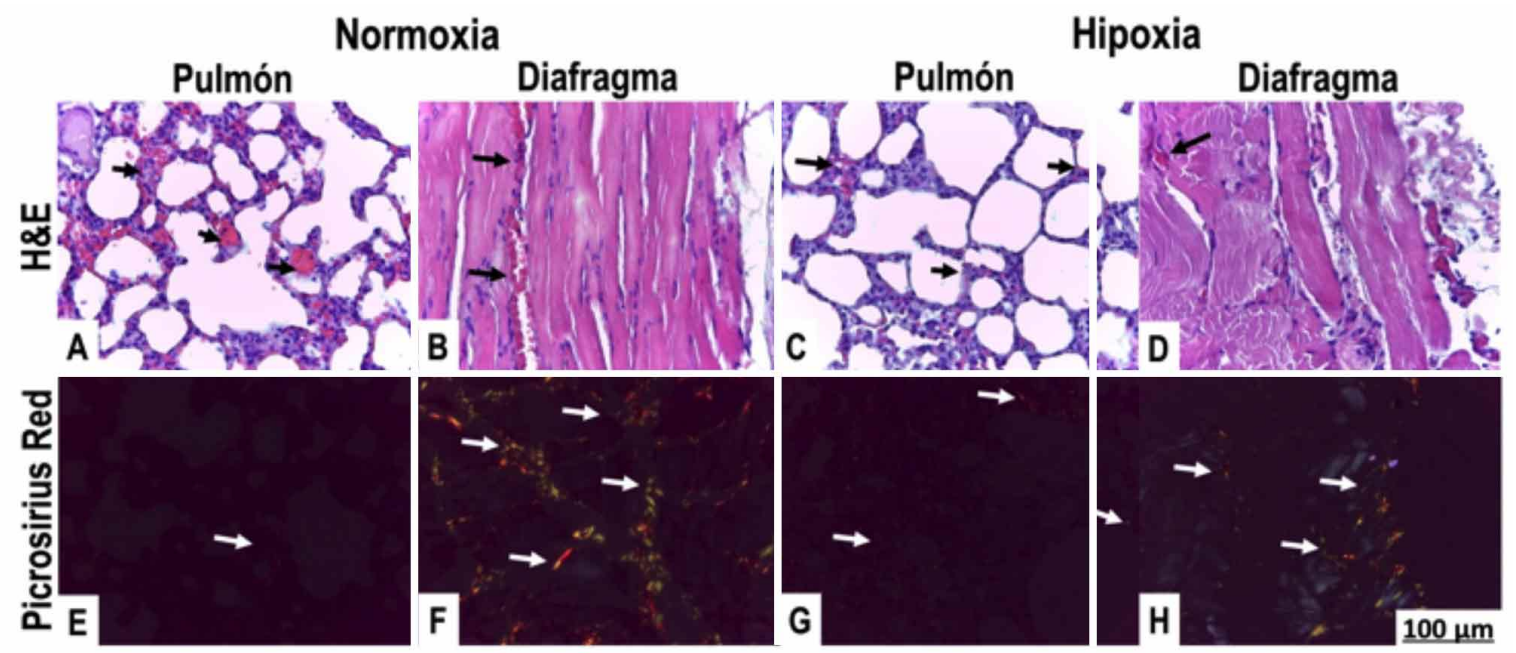

Fig 3. Comparación entre grupos normoxia e HHI en parénquima pulmonar. En A al D se observa tinción con H\&E, flechas indican la presencia de vasos sanguíneos. De la E al H muestras teñidas con Picrosirius Red, flechas indican la presencia de colágeno. 
$75,25 \pm 2,80 \%$ en el grupo HHI, además este último presentó una distribución irregular de las fibras musculares. El diafragma del grupo HHI soportó menos carga en relación al grupo con normoxia, se debería tal vez a un mayor volumen celular (miocitos) y mayor sostén (colágeno) del diafragma en normoxia.

Además de esto, el pulmón sometido a HHI, llegó a su deformación máxima con una menor tensión respecto al grupo control (Fig. 3). Este fenómeno se explicaría porque la hipoxia genera un edema pulmonar (Shukla et al., 2011). El engrosamiento de la pared arterial y su deterioro en el lecho vascular pulmonar, ocasionan que el parénquima pulmonar hipóxico pierda su punto de tensión máximo, provocando la acumulación de líquido extra en los espacios alveolares (Stenmark et al., 2006). También se ha determinado que el estrés inducido por hipoxia facilita el ascenso del tono vasomotor $y$ favorece a la vasoconstricción pulmonar. Ayudado por el aumento de moléculas que inducen cambios en el tono vascular, como factor de crecimiento endotelial vascular (VEGF), eritropoyetina (EPO) y endotelina-1 (ET-1) (Nehra et al., 2016). En relación ET-1, se ha visto como agente causal de las lesiones pulmonar, provocando daño patológico en el parénquima pulmonar a través de la modificación del tejido conectivo, y el resultante estado fibrótico (Ivy et al., 2001). En resumen, la evidencia presentada respalda la disminución en la deformación encontrada en ratas sometidas a HHI en este estudio.

Es importante destacar que los resultados encontrados están enmarcados en una experiencia previa, con un número pequeño de animales. Así, los principales hallazgos indican que el diafragma y el tejido pulmonar sometido a HHI sufren cambios estructurales en términos de tendencia, que se traducen en una disminución en su capacidad de resistir tensión.

AGRADECIMIENTOS. Los autores agradecen a CONICYT el soporte brindado por el proyecto Fondecyt 1170608 y a la DICYT de la Universidad de Santiago de Chile

CONEI, D.; DEL SOL, M.; MUÑOZ, R.; ESCOBAR, C. M.; SAAVEDRA, H.; HERRERA, E. A. \& GARCÍA-HERRERA, C. M. Morphological and mechanical effects in Sprague Dawley rats subjected to hypoxia cycles. Int. J. Morphol., 37(3):908-911, 2019.

SUMMARY: Extensive periods of hypoxic cause adaptive changes that make it possible to respond to the demands imposed by the environment. However, there are cases where this exposure is intermittent, as is the case of workers in andean areas. The objective of this communication was to verify the morphological and mechanical effects on diaphragm and lungs of rats subjected to intermittent hypoxic. Four 6-monthold Sprague Dawley rats were used. Two rats were subjected to 10 cycles of intermittent hypobaric hypoxic (IHH) of $96 \mathrm{~h} \mathrm{(} \mathrm{428} \mathrm{torr,} \mathrm{PO}_{2} 90 \mathrm{~mm}$ $\mathrm{Hg}$ ), followed by $96 \mathrm{~h}$ of normobaric normoxia, for 80 days. Uniaxial traction and staining tests were performed with Hematoxylin-Eosin and Picrosirius red de Junqueira. When comparing the curves of the diaphragms, those subjected to hypoxic slightly reduce their effort with respect to the condition of normoxia, in the lung tissue the hypoxic negatively affects its resistance, these show a lower slope with respect to the normoxics. In the histological analysis, the pulmonary parenchyma had a lower number of blood vessels and cellularity, such as a greater area fraction of alveolar spaces and amount of total collagen in IHH group. In the diaphragm, IHH group had a lower number of irregularly distributed myocytes and a lower amount of total collagen. In conclusion, the main findings indicate that the diaphragm and lung tissue subjected to IHH undergo structural changes, which result in a decrease in tensile strength.

\section{KEY WORDS: Hypoxic; Diaphragm; Lungs; Tension.}

\section{REFERENCIAS BIBLIOGRÁFICAS}

Adaos, C.; González, A.; Slater, D.; Medina, P.; Muñoz, R. \& Escobar, M. Análisis de presión inspiratoria máxima según tres protocolos en estudiantes voluntarios asintomáticos de la Universidad Católica del Maule, Chile. Rev. Chil. Enferm. Respir. 33:21-30, 2017.

Esteva, S.; Panisello, P.; Ramon Torrella, J.; Pages, T. \& Viscor, G. Enzyme activity and myoglobin concentration in rat myocardium and skeletal muscles after passive intermittent simulated altitude exposure. J. Sports. Sci. 27(6):633-40, 2009

Herrera, E. A.; Farías, J. G.; González-Candia, A.; Short, S. E.; Carrasco-Pozo, C. \& Castillo, R. L. $\Omega 3$ Supplementation and intermittent hypobaric hypoxia induce cardioprotection enhancing antioxidant mechanisms in adult rats. Mar. Drugs. 13(2):838-60, 2015.

Ivy, D.; McMurtry, I. F.; Yanagisawa, M.; Gariepy, C. E.; Le Cras, T. D.; Gebb, S. A.; Morris, K. G.; Wiseman, R. C. \& Abman, S. H. Endothelin B receptor deficiency potentiates ET-1 and hypoxic pulmonary vasoconstriction. Am. J. Physiol. Lung Cell. Mol. Physiol., 280(5):1040-8, 2001.

Kuthe, C. D. \& Uddanwadiker, R. V. Investigation of effect of fiber orientation on mechanical behavior of skeletal muscle. J. Appl. Biomater. Funct. Mater. 14(2):154-62, 2016

Lewis, P. \& O'Halloran, K. D. Diaphragm Muscle Adaptation to Sustained Hypoxia: Lessons from Animal Models with Relevance to High Altitude and Chronic Respiratory Diseases. Front. Physiol., 12(7):623, 2016.

McMorrow, C.; Fredsted, A.; Carberry, J.; O'Connell, R. A.; Bradford, A.; Jones, J. F. \& O'Halloran, K. D. Chronic hypoxia increases rat diaphragm muscle endurance and sodium-potassium ATPase pump content. Eur. Respir. J., 37(6):1474-81, 2011.

Mortola, J. P. How to breathe? Respiratory mechanics and breathing pattern. Respir Physiol. Neurobiol., 261:48-54, 2019.

Nehra, S.; Bhardwaj, V.; Bansal, A. \& Saraswat, D. Nanocurcumin accords protection against acute hypobaric hypoxia induced lung injury in rats. J. Physiol. Biochem., 72(4):763-79, 2016.

Panisello, P.; Torrella, J. R.; Esteva, S.; Pagés, T. \& Viscor, G. Capillary supply, fibre types and fibre morphometry in rat tibialis anterior and diaphragm muscles after intermittent exposure to hypobaric hypoxia. Eur. J. Appl. Physiol., 103(2):203$13,2008$.

Shukla, D.; Saxena, S.; Purushothaman, J.; Shrivastava, K.; Singh, M.; Shukla, S. Malhotra, V. K.; Mustoori, S. \& Bansal, A. Hypoxic preconditioning with cobalt ameliorates hypobaric hypoxia induced pulmonary edema in rat. Eur. J. Pharmacol., 656(1-3):101-9, 2001

Stenmark, K. R.; Fagan, K. A. \& Frid, M. G. Hypoxia-induced pulmonary vascular remodeling: cellular and molecular mechanisms. Circ. Res., 99(7):675-91, 2006.

Urrutia, A. A. \& Aragonés, J. HIF Oxygen Sensing Pathways in Lung Biology. Biomedicines, 6(2):1-10, 2018.

Zhu, X.; Heunks, L. M.; Versteeg, E. M.; Van der Heijden, H. F.; Ennen, L.; Van Kuppevelt, T. H.; Vina, J. \& Dekhuijzen, P. N. Hypoxia-induced dysfunction of rat diaphragm: role of peroxynitrite. Am. J. Physiol. Lung. Cell. Mol. Physiol., 288(1):16-26, 2005.

\section{Dirección para correspondencia:}

Daniel Conei Valencia

Programa de Doctorado en Ciencias Morfológicas

Universidad de La Frontera

Temuco - CHILE

Recibido : 18-03-2019

Aceptado: 16-04-2019

Email: d.conei01@ufromail.cl 